\title{
The potassium channel Kcne3 is a VEGFA-inducible gene selectively expressed by vascular endothelial tip cells
}

\author{
Ron A. Deckelbaum ${ }^{1}$ (1) Ivan B. Lobov ${ }^{6}$ Eunice Cheung ${ }^{2} \cdot$ Gabor Halasz $^{4} \cdot$ Saathyaki Rajamani ${ }^{1}$. Julia Lerner ${ }^{1}$. \\ Chunxiang Tong ${ }^{1} \cdot{\text { Zhe } \mathrm{Li}^{3}}^{3}$ Patricia Boland ${ }^{3} \cdot$ Melissa Dominguez ${ }^{1} \cdot$ Virginia Hughes $^{1} \cdot$ George D. Yancopoulos ${ }^{5}$. \\ Andrew J. Murphy ${ }^{5}$. Gavin Thurston ${ }^{3} \cdot$ Jingtai $\mathrm{CaO}^{2} \cdot$ Carmelo Romano $^{2} \cdot$ Nicholas W. Gale $^{1}$
}

Received: 24 July 2019 / Accepted: 2 November 2019 / Published online: 21 November 2019

(c) The Author(s) 2019

\begin{abstract}
Angiogenesis is largely driven by motile endothelial tip-cells capable of invading avascular tissue domains and enabling new vessel formation. Highly responsive to Vascular Endothelial Growth-Factor-A (VEGFA), endothelial tip-cells also suppress angiogenic sprouting in adjacent stalk cells, and thus have been a primary therapeutic focus in addressing neovascular pathologies. Surprisingly, however, there remains a paucity of specific endothelial tip-cell markers. Here, we employ transcriptional profiling and a lacZ reporter allele to identify Kcne3 as an early and selective endothelial tip-cell marker in multiple angiogenic contexts. In development, Kcne3 expression initiates during early phases of angiogenesis (E9) and remains specific to endothelial tip-cells, often adjacent to regions expressing VEGFA. Consistently, Kcne 3 activation is highly responsive to exogenous VEGFA but maintains tip-cell specificity throughout normal retinal angiogenesis. We also demonstrate endothelial tip-cell selectivity of Kcne3 in several injury and tumor models. Together, our data show that Kcne3 is a unique marker of sprouting angiogenic tip-cells and offers new opportunities for investigating and targeting this cell type.
\end{abstract}

Keywords Endothelial tip-cell $\cdot$ Kcne $3 \cdot$ VEGFA $\cdot$ Retinal angiogenesis

Ron A. Deckelbaum and Ivan B. Lobov have contributed equally to this study

Electronic supplementary material The online version of this article (https://doi.org/10.1007/s10456-019-09696-8) contains supplementary material, which is available to authorized users.

Ron A. Deckelbaum

ron.deckelbaum@regeneron.com

1 Department of Pre-Therapeutic Target Discovery, Regeneron Pharmaceuticals Inc, Tarrytown, NY 10591, USA

2 Department of Ophthalmology, Regeneron Pharmaceuticals Inc, Tarrytown, NY 10591, USA

3 Department of Oncology \& Angiogenesis, Regeneron Pharmaceuticals Inc., Tarrytown, NY 10591, USA

4 Department of Bioinformatics, Regeneron Pharmaceuticals Inc, Tarrytown, NY 10591, USA

5 Department of Research, Regeneron Pharmaceuticals Inc, Tarrytown, NY 10591, USA

6 Palmira Biopharma, Moscow 143026, RF, Russia

\section{Introduction}

New blood vessel formation, or neovascularization, is a necessary physiological process that is highly coordinated with tissue growth and homeostasis, but is frequently dysregulated in disease states ranging from cancer to ischemia [1-3]. Angiogenesis, the primary mode of neovascularization, ensues through selection and sprouting of migratory endothelial cells (ECs) that break away from their stable positions within pre-existing blood vessels to form lumenized tubules that further remodel into an elaborate network of arteries, veins and capillaries [4, 5]. Residing at the leading edge of angiogenic sprouts, specialized endothelial tip cells (ETCs) retain migratory and invasive properties that allow for sprouts to reach the avascular tissue environment. ETCs express abundant levels of vascular endothelial growth-factor-A receptor 2 (VegfR2), and are highly responsive to the pro-migratory effects of VEGFA produced by tissues under oxygen and nutrient depravation [6]. Trailing behind ETCs are phenotypically distinct endothelial stalk cells (ESCs). Mediating vessel lumenization, ESCs are highly proliferative but exhibit reduced 
VEGFA-responsiveness and strongly express the Delta-like 4 (Dll4) receptor Notch1 [7, 8]. In a negative feedback loop, VEGFA stimulation of Dll4 expression in ETCs results in Notch1 activation in subjacent ESCs, which in turn downregulate VegfR2 and promote pericyte recruitment and vessel maturation [9-11]. The balance between ETCs and ESCs is therefore of crucial importance to developmental and pathogenic angiogenesis, where even partial loss of VEGFA or Dll4 function results in blunted angiogenesis or exuberant endothelial sprouting, respectively [12-14]. Despite the fact ETCs have been a primary focus for targeted antiangiogenic therapies in tumors and vascular eye disease, there remains a surprising paucity of specific molecular markers for this cell type.

Distinguishing ETCs has historically relied on studies of retinal angiogenesis, where a vascular plexus develops from the central optic stalk that expands radially and superficially along the retinal inner neural surface (P0-P7 in mouse, [15]). This unique concentric planar organization allows for spatial localization of ETCs at the leading edge of the vascular plexus, where elaborate filopodia extensions distinguishes them morphologically. Current molecular identifiers of ETCs include the endothelial markers $K d r$ (VegfR2), Flt4, $P d g f b, D l l 4$, and $U n c 5 b$, which exhibit higher expression levels relative to ESCs but are not exclusive to ETCs $[6,8$, 16-18]. For example, Dll4 and Unc5b are expressed by both ETCs and by arterial endothelial cells, while Flt4 is also expressed by venous endothelial cells-reflective of their functional roles in sprouting angiogenesis and vessel remodeling. More recently, interrogation of expression profiles from isolated retinal ECs led to the identification of ETCenriched genes (e.g. Cxcr4, Apln, Esm1, and Angpt2), with the VEGFA-response gene Esml validated by genetic fate mapping [19-23]. However, a number of these putative ETC markers are also active in other vascular compartments, and it is therefore unclear whether they confer ETC-specificity outside of retinal angiogenesis. Thus, the characterization of distinct ETC markers within multiple angiogenic contexts remains a pertinent objective.

KCNE3, formerly named Mink-related Peptide 2 (MiRP2), is a member of the KCNE family of ancillary $\beta$-subunits that assemble with $\alpha$-subunits of voltage-gated potassium $\left(\mathrm{K}^{+}\right)$ion channels, including KCNQ1 and Kv3.4, and which functions in modulating their electrophysiological properties [24, 25]. In mice, Kcne3 is reportedly expressed in the small intestine, colon, and trachea, where it localizes to the basolateral aspects of the mucosal epithelium [26]. Interestingly, Kcne 3 knockout mice are viable and fertile but exhibit perturbations in transepithelial transport of $\mathrm{Cl}^{-}$and cardiac arrhythmias associated with adrenal-targeted autoimmune responses [26, 27]. However, expression of Kcne3 in vascular endothelial cells has not been described. In the present study we interrogated transcriptomes from early postnatal mouse retinas subjected to transient VEGFA activation or blockade and reveal Kcne 3 as a robust endothelialspecific VEGFA-inducible gene. Utilizing a Kcne3-lacZ knock-in reporter mouse line, we show that, in vivo, Kcne 3 activation initiates by ETCs within multiple embryonic vascular plexuses (E9.5-E11.5), in close association with domains of VEGFA expression. Kcne3 is not exclusively expressed in the vasculature: At later developmental stages and in the adult Kcne 3 is also expressed in the gut and tracheal epithelium.

Our studies provide first evidence that Kcne 3 may be exploited as a general ETC marker in multiple angiogenic contexts and opens new opportunities for targeting this specialized endothelial cell type for addressing neovascular disease states.

\section{Materials and methods}

\section{Animal care and anesthesia}

All animal studies were approved and performed in accordance with Regeneron's Institutional Animal Care and Use Committee (IACUC) guidelines. For survival corneal surgeries, pups were anaesthetized using a mixture of oxygen and 2 volumes (\%) of isoflurane employing the VETequip vaporizer. Local anesthesia to the eye was performed by applying a single drop of proparacaine.

\section{Intravitreal administration of VEGFA and VEGF-Trap}

Intravitreal (ITV) microinjections (50-500 nl) were made between the equator and the corneal limbus using a Drummond nanoinjector equipped with a glass needle as described [9]. For this study, intravitreal injections were performed on 6-day old pups (P6), which received $4 \mu \mathrm{g}$ of hFc (REGN379), $4 \mu \mathrm{g}$ of VEGF-Trap (REGN3), or $1.5 \mu \mathrm{g}$ of VEGFA 165 (REGN110) per eye. The following day (P7), retinas were dissected and used for RNAseq or ISH/IB4 analyses.

\section{cRNAseq transcriptome analysis}

Total RNA was purified using the MagMAX-96 for Microarrays Total RNA Isolation kit (Ambion) according to the manufacturer's instructions, in which genomic DNA was removed using MagMAXTurboDNase buffer and Turbo DNase. mRNA was purified from total RNA using the Dynabeads mRNA purification kit (Invitrogen) according to the manufacturer's instructions. For the P8 retina profiling, strand-specific RNA sequencing (RNA-Seq) libraries were prepared using the ScriptSeq mRNA-Seq Library Preparation kit (Epicentre). Twelve-cycle PCR was performed to 
amplify libraries. For the OIR study, cDNA was synthesized and amplified (12-cycle PCR) from ten nanogram total RNA using SMARTer ${ }^{\circledR}$ Ultra ${ }^{\circledR}$ Low RNA Kit (Clontech). Nextera XT library prep kit (Illumina) was used to generate the final sequencing library (12 PCR cycles performed to amplify libraries) using $1 \mathrm{ng}$ of cDNA as the input. Sequencing was performed on an Illumina HiSeq 2000 instrument by a multiplexed, single-read run with 33 cycles. Raw sequence data (BCL files) were converted to Fastq format via Illumina Casava 1.8.2. Reads were decoded based on their barcodes, and read quality was evaluated using Fastqc (www. bioinformatics.babraham.ac.uk/projects/fastqc/). Reads were mapped to the mouse transcriptome (NCBI genome assembly GRCm $38 / \mathrm{mm} 10$ ) using ArrayStudio's OSA aligner, allowing for two mismatches. Exon mapped reads were summed at the gene level. Genes differentially expressed between hFc-, VEGFA-, or VEGF-Trap-treated samples were obtained using DeSeq2. A gene was considered regulated in a particular comparison if the nominal $P$ value from DeSeq2 was less than 0.01 and if the mean expression increased or decreased by at least $50 \%$.

\section{Kcne3-lacZ reporter mice}

Employing Velocigene technology [28], a BAC construct was engineered to replace the entire coding sequence of the murine Kcne 3 gene with a $\beta$-galactosidase (lacZ)/ floxed-neomycin cassette, inserted in-frame at the initiating ATG within exon 4. Homologous recombination within F1H4 ES cells (C57BL6/129SvJ hybrid), was followed by identification of correctly targeted ES cell clones using the Loss-Of-Allele Assay (LOA), [28]. Two independent ES clones were employed to generate chimeric mice that were subsequently bred to C57BL6 females to generate $F_{1}$ mice, which were genotyped by LOA and verified histochemically for $\beta$-galactosidase activity. Heterozygous Kcne3-lacZ mice were maintained on a mixed genetic background (75\% C57BL6/25\% 129SvJ).

\section{LacZ reporter analysis}

LacZ staining of whole embryos and retina was performed as previously described [29]. Briefly, dissected samples were fixed in $2 \%$ paraformaldehyde $/ 0.2 \%$ glutaraldehyde for $1 \mathrm{~h}$ on ice, washes in PBS, and incubated with X-gal staining solution $\left(5 \mathrm{mM} \mathrm{K}_{3} \mathrm{Fe}(\mathrm{CN})_{6}, 5 \mathrm{mM} \mathrm{K}_{4} \mathrm{Fe}(\mathrm{CN})_{6}, 2 \mathrm{mM}\right.$ $\mathrm{MgCl}_{2}, 0.01 \%$ deoxycholate, $0.02 \%$ igepal, $1 \mathrm{mg} / \mathrm{ml} \mathrm{X-gal)}$ for $12 \mathrm{~h}$ at $37^{\circ} \mathrm{C}$. Following post-fixation with $2 \%$ paraformaldehyde, specimens were washed in PBS and transferred into $70 \%$ glycerol/PBS prior for imaging. For histological preparations, samples were fixed as above, sunk into $15 \%$ and $30 \%$ sucrose, and OCT-embedded for cryosectioning. Following brief post-fixation, sections were stained with
X-gal solution and counterstained with Neutral Red prior to mounting with DPX mounting media.

\section{Whole mount In Situ Hybridization and isolectin staining of retinas}

A riboprobe encompassing 557 base pairs of the murine Kcne 3 coding sequence was amplified by reverse transcriptase (RT)-PCR (iScript cDNA Synthesis Kit, BioRad), employing the following primers: 5'-GAG ACT TCC AAC GGG ACT GA-3', and 5'-CGC CAC AGC TTC CTC TTC$3^{\prime}$. This was subsequently cloned into the pGEMT-Easy vector (Promega), linearized with SacII, and mRNA transcription performed with SP6 polymerase and digoxigeninlabeled UTP using the MEGAscript kit (Ambion). Dissected retinas were fixed in $4 \%$ paraformaldehyde (PFA)/PBS for $16 \mathrm{~h}$ at $4{ }^{\circ} \mathrm{C}$, gradually dehydrated into methanol and then rehydrated into PBS. Tissues were digested with proteinase $\mathrm{K}(10 \mu \mathrm{g} / \mathrm{ml})$ for $20 \mathrm{~min}$, and incubated in buffered detergent (1\% NP-40, $1 \%$ SDS, $0.5 \%$ Na deoxycholate, 1 mM EDTA, $150 \mathrm{mM} \mathrm{NaCl}, 50 \mathrm{mM}$ Tris, pH 8.0) for $30 \mathrm{~min}$. Following post-fixation in $0.2 \%$ glutaraldehyde $/ 4 \%$ PFA, retinas were placed in hybridization buffer ( $50 \%$ formamide, $5 \times \mathrm{SSC}, 1 \%$ SDS, $50 \mu \mathrm{g} / \mathrm{ml}$ heparin, $200 \mu \mathrm{g} / \mathrm{ml}$ BSA, $100 \mu \mathrm{g} / \mathrm{ml}$ yeast RNA) for $60 \mathrm{~min}$ at $65^{\circ} \mathrm{C}$, and then overnight under similar conditions with the addition of Kcne 3 riboprobe. Unbound probe was removed by successive incubations with wash buffer (50\% formamide, $1 \times$ SSC, $0.1 \%$ Tween 20 ) and a $1: 1$ mix of wash buffer-MABT (100 mM maleic acid pH 7.5, $150 \mathrm{mM} \mathrm{NaCl}, 0.1 \%$ Tween 20 ) at $65^{\circ} \mathrm{C}$. Following additional washes with MABT at room temperature, specimens were incubated with $15 \%$ normal goat serum/MABT for $2 \mathrm{~h}$ and then overnight with AP-conjugated anti-DIG (Roche, 1:5000). Following repeated washing with MABT, signal was detected using BM-Purple reagent (Roche). For subsequent vessel labeling, retinas were treated with biotinylated isolectin (Vector, \#B1205; 1:200/PBST), washed several times in PBST and then with TBTI $(50 \mathrm{mM}$ Tris-HCl, $\mathrm{pH}$ $8.0,150 \mathrm{mM} \mathrm{NaCl}, 0.1 \%$ Tween-20, $10 \mathrm{mM}$ imidazole, $0.2 \%$ BSA). Fluorescent signal was detected using Cy3-tyramide (Perkin Elmer, \#NEL744001KT) and $0.0015 \%$ hydrogen peroxide for $1 \mathrm{~h}$.

\section{In Situ Hybridization by RNAscope on histological sections}

Embryonic tissues were fixed in 4\% PFA/PBS for $16 \mathrm{~h}$ and cryopreserved in OCT. Simultaneous detection of Pecam 1 and Kcne 3 mRNA was performed according to protocols developed by the manufacturer-Advanced Cell Diagnostics [30], using the following probes: Mm-Kcne3-C1, cat\# 493831 and Mm-Pecam1-C2, cat\#316721. Following all hybridization steps tyramide- 488 amplification was 
A

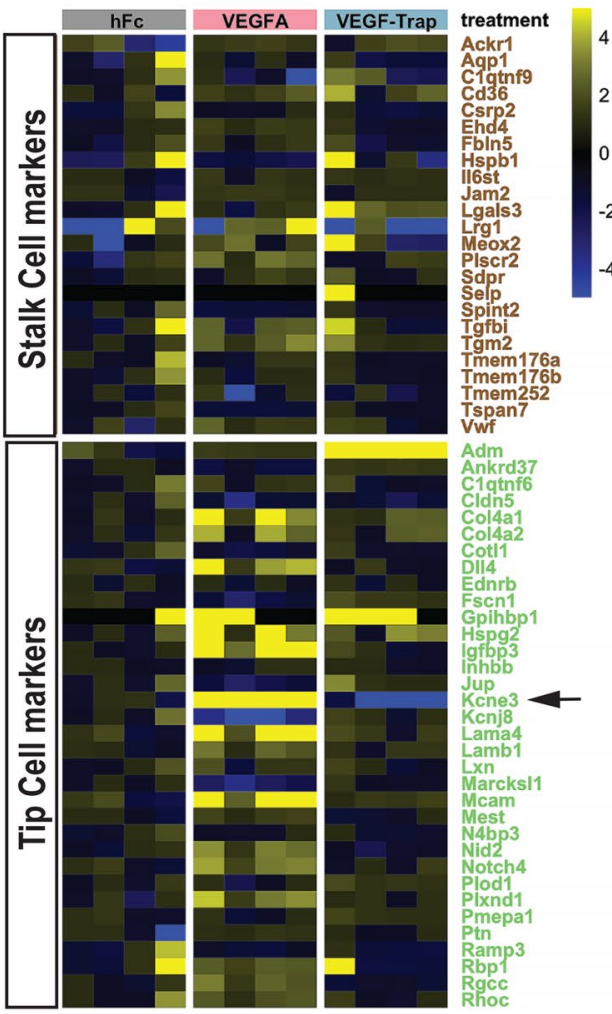

B

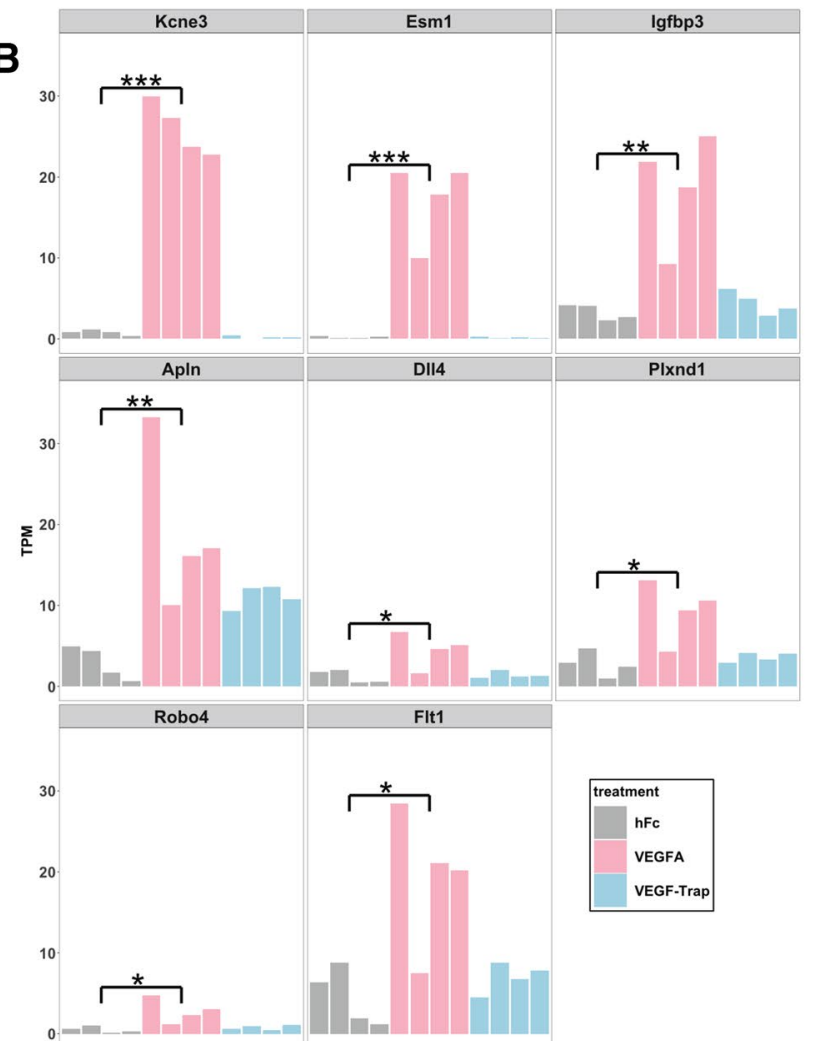

C
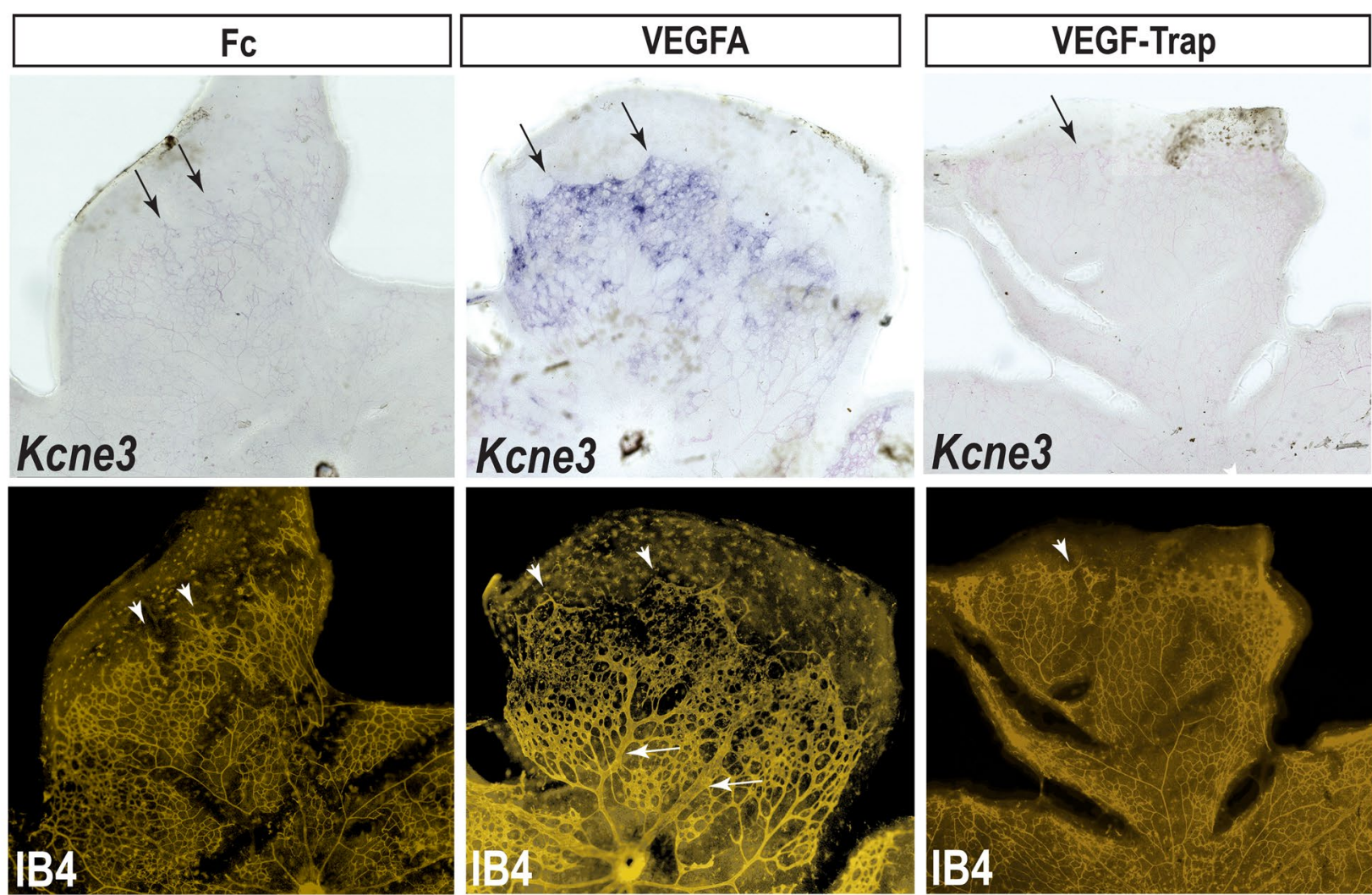

Kcne3

*. Kcne3
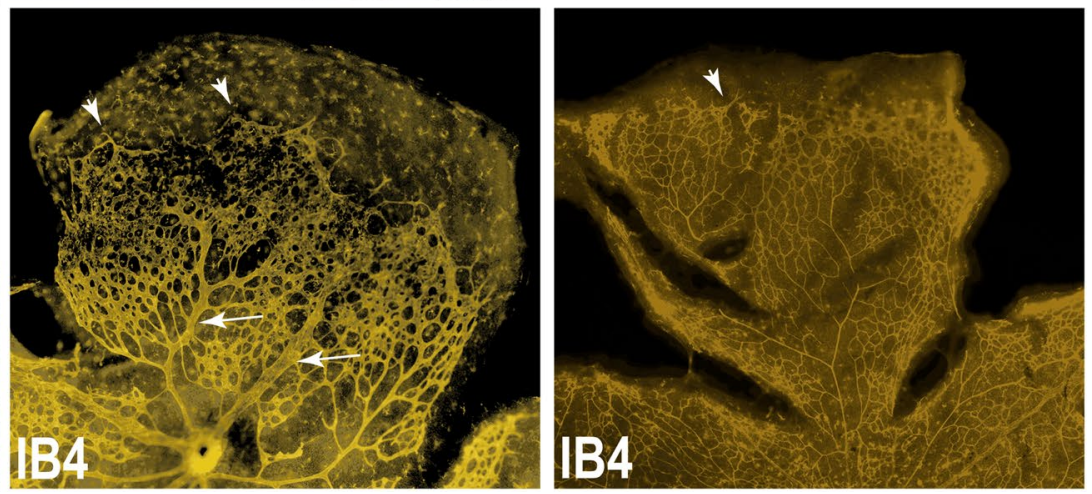
4Fig. 1 Kcne3 is transcriptionally regulated by VEGFA in retinal endothelial cells. a Heat map displaying the expression profiles of previously defined endothelial stalk-cell or tip-cell genes, in postnatal day 8 (P8) mouse retinas 24-h following intravitreal injection of $\mathrm{hFc}$, VEGFA, or VEGF-Trap. Scale shown is $\log _{2}$ transformed fold-change, relative to the median of $\mathrm{hFc}$-treated controls. The analysis of $N=4$ mice per treatment group is shown. b Comparative expression of Kcne3 to cognate endothelial and ETC genes-Esm1, Igfbp3, Apln, Dll4, Plxnd1, Robo4, and Flt1, in retinas exposed to $\mathrm{hFc}$, VEGFA, or VEGF-Trap. $* * * \mathrm{P}_{\mathrm{BH} \text {-corrected }}=8 \times 10^{-40}-5 \times 10^{-38}$, $* * \mathrm{P}_{\mathrm{BH} \text {-corrected }}=4.6 \times 10^{-10}-1.7 \times 10^{-7}, \quad * \mathrm{P}_{\text {BH-corrected }}=6.5 \times 10^{-4}-2$ $.8 \times 10^{-3}$. c Top panel: whole mount ISH analysis of Kcne3 mRNA expression in retinal preparations from P7 mice, 24-h following intravitreal injection of hFc, VEGFA, or VEGF-Trap (representative samples from $N=4$ mice per treatment group). Weak Kcne 3 signal is observed in ETCs and ESCs within the leading angiogenic front of $\mathrm{hFc}$-injected eyes (left panel, arrows), but is dramatically increased in VEGFA-injected eyes (middle panel, arrows). By comparison, no Kcne3 signal is observed in VEGF-Trap-injected eyes (right panel). Lower panel: sequential fluorescent isolectin (IB4-cy3) staining reveals significant vessel dilation in VEGFA-injected eyes (middle panel; arrows), and also shows that the majority of Kcne3+ cells localize to the peripheral aspects of the vascular plexus (middle panels, arrow and arrowheads)

employed to detect specific Kcne3 signal, while Pecaml was detected using the RNAscope ${ }^{\circledR}$ 2.0 HD Detection Kit (RED).

\section{Immunofluorescence on histological sections}

Frozen OCT-embedded sections of mouse embryos were blocked for $1 \mathrm{~h}$ with $10 \%$ normal goat serum/PBST, incubated for $16 \mathrm{~h}$ at $4{ }^{\circ} \mathrm{C}$ with antibodies to $\beta$-galactosidase (Abcam, ab9361) and Pecam1 (BD Pharmingen, 553370). Immunoreactivity was determined using horseradish peroxidase conjugated donkey anti-chicken $\mathrm{F}(\mathrm{ab})_{2}$ (Jackson Immunolabs, 703-036-155) and donkey anti-rat (Jackson Immunolabs, 712-036-153), followed by sequential cy3-tyramide and 488-tyramide signal amplification.

\section{Oxygen-induced retinopathy (OIR) model}

OIR was produced following the method developed by Smith et al. [31]. Briefly, litters of 6-day old (P6) mouse pups and their dams were placed in hyperoxia ( $75 \%$ oxygen) to induce capillary obliteration in the central retina. Elevenday old pups (P11) were returned to room air and analyzed immediately or at P16. In a second study, P11 pups were subjected to normoxia until $\mathrm{P} 15$, at which time they received intraperitoneal injections of hFc or VEGF-Trap at $25 \mathrm{mg} / \mathrm{kg}$. Retinas from the right eyes of these mice were assessed by fluorescein Griffonia Simplicifolia I (GS lectin I) (Vector Laboratories) staining of vessels and by NG2 (Millipore) with Alexa-Fluor 594 (Invitrogen) staining of neovascular tufts. Retinas from the left eyes were assessed by RNAseq transcriptomics 6- and 24-h following treatment $(N=4-5$, per group).

\section{Corneal suture model}

Employing adult Kcne3-lacZ mice, corneal sutures were placed in proximity to the central part of the cornea as previously described [32]. After 9 days, mice were euthanized and whole eyes were fixed in $4 \%$ PFA, washed in PBS, and stained in X-gal staining solution. Corneas were then subdissected, flat mounted, and imaged by light microscopy.

\section{LCC tumor model}

Lewis Lung carcinoma cells $\left(5 \times 10^{5}\right)$ were injected under the dorsal skin of syngeneic adult heterozygous or homozygous Kcne3-lacZ mice. Seven to ten days postimplantation a palpable tumor could be visualized under the skin at the injection site, at which time mice were euthanatized and tumors were processed for X-gal staining and imaging.

\section{ES tumor model}

F1H4 ES cells were implanted sub-cutaneously into SCID mice as previously described [33]. Tumor growth was allowed to proceed until reaching $300-500 \mathrm{~mm}^{3}$, after which these were dissected, fixed in $4 \%$ paraformaldehyde, and processed for whole mount or histological X-gal staining. Representative sections were also stained for immunohistochemical detection of CD31 (Pecam1).

\section{Results}

\section{Kcne3 is induced by VEGFA in retinal vascular endothelial cells}

We have previously shown that localized delivery of VEGFA to the developing postnatal retina rapidly and profoundly alters vessel morphogenesis, during which endothelial cells acquire a distinct transcriptional profile [34]. To better characterize the VEGFA-regulated endothelial transcriptome in vivo, we profiled by RNA-seq postnatal day 8 (P8) retinas $24 \mathrm{~h}$ following intravitreal administration of recombinant VEGFA $_{165}$, human IgG1 $(\mathrm{hFc})$, or VEGF-Trap $(N=4$, per treatment group). A composite of VEGFR1 and VEGFR2 Ig domains fused to human Fc, VEGF-Trap effectively binds and neutralizes the activity of VEGFA, VEGFB, and placental-growth factor (PLGF) [35]. Compared to retinas from eyes injected with $\mathrm{hFc}$ protein, retinas exposed to VEGFA exhibited a robust signature consisting of 742 upregulated and 1280 downregulated genes (Fig. 1a, b and Supplemental Table 1), whereas VEGF-Trap injection resulted in 93 upregulated and 71 downregulated genes. To further examine VEGFA-induced effects on ETCs, we evaluated the expression of defined ETC and ESC signature markers 

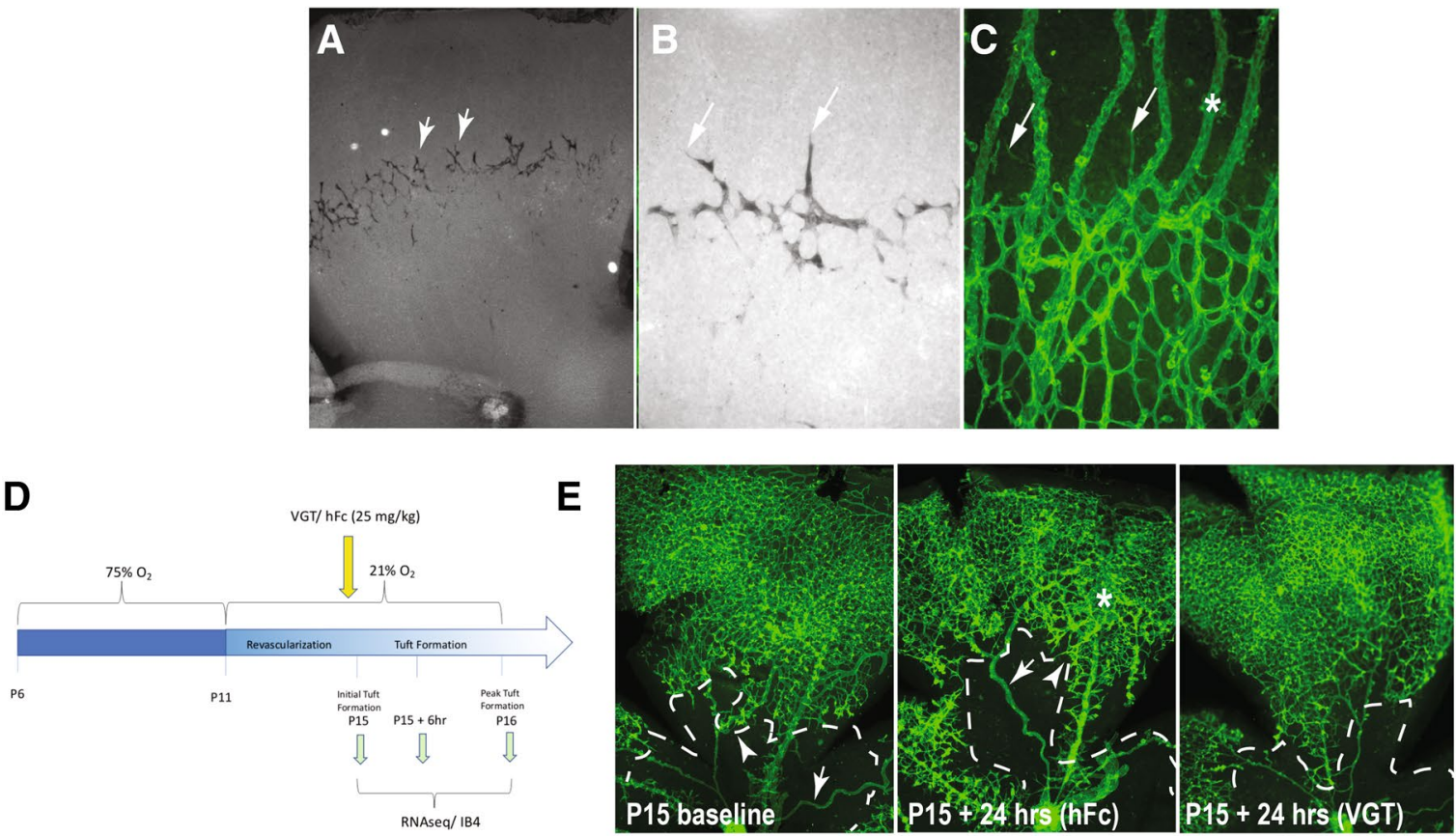

F
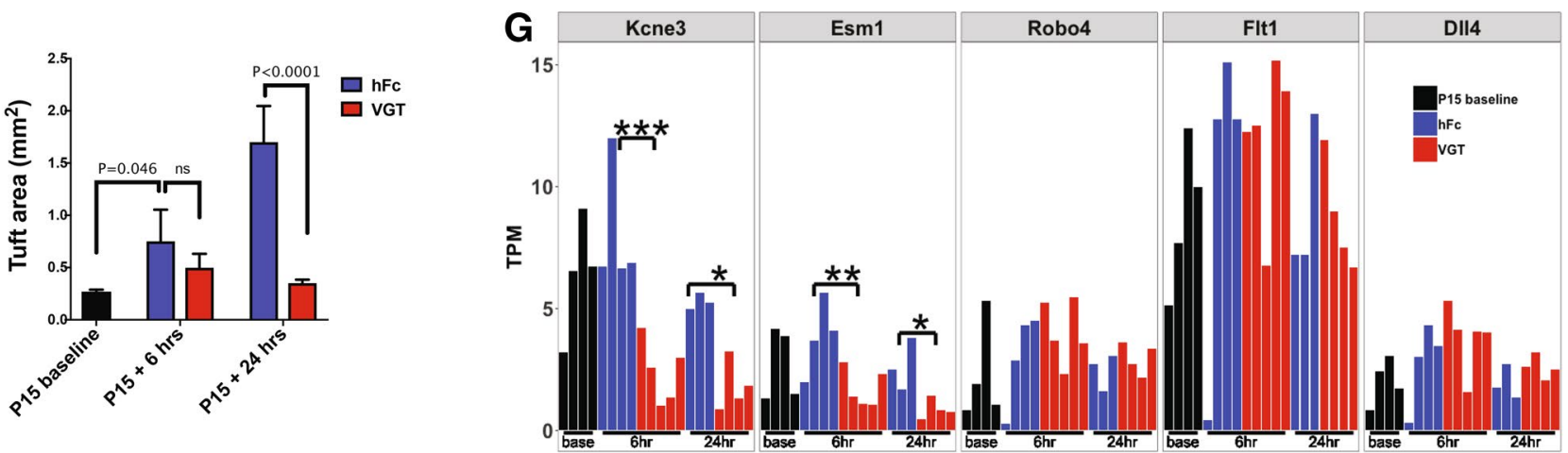

H
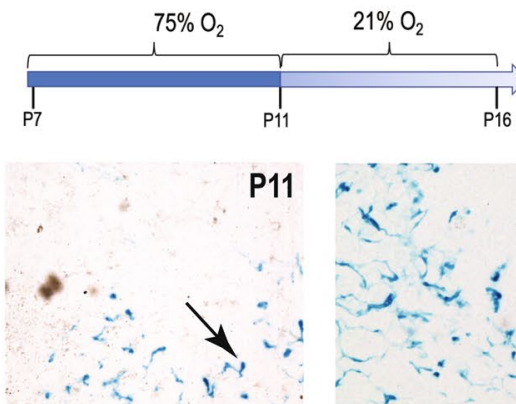

Kene3-lacz
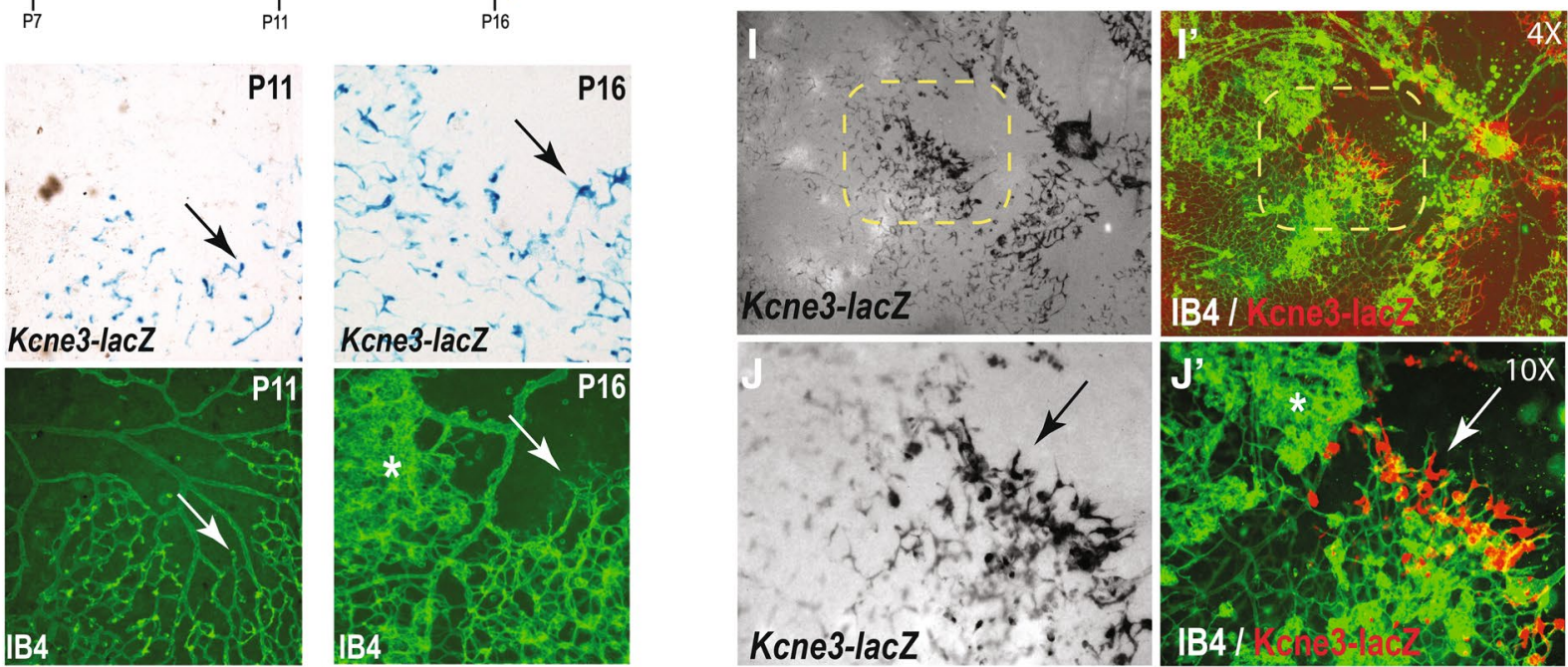
4Fig. 2 Kcne3 is activated in ETCs during normal and pathogenic retinal angiogenesis. a-c $\beta$-galactosidase staining of P7 retina from heterozygous Kcne3-lacZ reporter mice at low and high-power showing specific expression in ETCs and weaker expression in ESCs along the angiogenic front (arrows a, b). c Comparative fluorescent isolectin (IB4) showing the overall vascular pattern compared to that in b; staining shows that Kcne3-lacZ is absent from hyaloid vessels (asterisk), but labels endothelial cells at the vascular front. d Schematic illustration of regimen for short-term OIR experiments. At the end of the hyperoxic phase (P11), single dose intravitreal injections of $\mathrm{hFc}$ or VEGF-Trap (VGT) was performed. Retinal samples were analyzed at baseline, 6-h, and 24-h from the start of neovascular tuft formation at P15 in normoxia. For each independent time point, analysis was performed on $N=3-5$ mice per treatment group. e IB4labeled retinal vessels at baseline and 24-h after return to normoxia, showing a reduction in the vaso-obliterative area (dotted line) and in neovascular tuft formation (asterisk) following VEGF-Trap delivery. f Quantitative image analysis of neovascular tuft area at baseline, 6-, and 24-h following return to normoxia. g Relative mRNA expression of selected endothelial markers, expressed as transcripts per million (TPM), derived from an RNAseq analysis of retinas subjected to OIR. Downregulation in expression levels of Kcne 3 and Esm 1 are observed under VEGF-Trap treatment conditions (compared to $\mathrm{hFc}$ ), but other EC-specific genes are not significantly affected. $\mathbf{h}$ Analysis of Kcne3-lacZ reporter expression under standard OIR conditions (schematic). Comparative lacZ- and IB4-stained retina highlights the detection of Kcne3 reporter activity in putative ETCs bordering the avascular zone that forms following a 4-day exposure to $75 \% \mathrm{O}_{2}$, and which intensifies during 5-days upon return to normoxia (arrows). Note the absence of lacZ + cells in neovascular tufts (asterisk). i, j' Low and high-power images of Kcne3-lacZ P16 retina, 5-days following the return to normoxia, detecting $\beta$-galactosidase and IB4+ vessels. Kcne3-lac ${ }^{+}$ETCs inundate the vascular-avascular interface (arrows), but are largely absent from neovascular tufts (asterisk). $* * * \mathrm{P}_{\text {value }}=1.2 \times 10^{-5}, * * \mathrm{P}_{\text {value }}=6.4 \times 10^{-5}, * \mathrm{P}_{\text {value }}=6.5 \times 10^{-4}$

derived from prior single-cell RNA-seq analyses of tumors [36]. Interestingly, many of the tumor-derived ETC markers were upregulated in retinas following transient VEGFA treatment, whereas ESC markers were largely unperturbed (Fig. 1a). Notably, VEGFA treatment resulted in the activation of previously reported ETC genes_Esm1, Igfbp3, Dll4 [7, 19, 20]. Conspicuously, the retinal expression of one gene, Kcne3, closely mimicked that of the VEGFAresponse gene $E s m 1$, where a dramatic 30-fold increase was observed following treatment with VEGFA (Fig. 1b). However, unlike most other putative ETC and generic EC genes that were upregulated by VEGFA (e.g. Apln, Dll4, Plxnd1, Flt1), the basal level of Kcne3 was attenuated by VEGF-Trap, suggesting that Kcne 3 is a VEGFA-response gene in endothelial cells (Fig. 1a, b, supplemental Table 1).

We next sought to identify the cellular source of Kcne3 mRNA by performing whole mount In Situ Hybridization (ISH) on retinas prepared $24 \mathrm{~h}$ after intravitreal delivery of VEGFA, VEGF-Trap, or hFc $(N=3-4)$. Chromogenic staining resulting from hybridization of the Kcne3 riboprobe was examined in retinas co-labeled with isolectin-B4 (IB4), an agent used to specifically detect vascular endothelial cells [9]. Examination of hFc-injected eyes showed weak Kcne3 signal co-localizing with IB4-positive ECs within the leading edge of the vascular plexus (Fig. 1c, left panels), but no signal was detected within the neural layers of the retina. Remarkably, VEGFA exposure dramatically increased the level of Kcne3 expression within the vascular plexus, where strong staining was observed in both ETCs and ESCs but not in established arteries or veins (Fig. 1c, center panels). By comparison, Kcne3 mRNA was not detected in retinas following delivery of VEGF-Trap (Fig. 1c, right panels). Our data therefore suggests that Kcne3 is highly activated by VEGFA in retinal endothelial cells, prompting us to further investigate its specificity to ETCs.

\section{Kcne3 is an ETC-specific gene during retinal angiogenesis}

To better characterize endogenous Kcne3 expression during normal phases of retinal development, we employed homologous recombination (VelociGene technology) to generate genetically modified mice expressing $\beta$-galactosidase (lacZ) in place of the Kcne3 coding sequence (Suppl. Fig. 1). Mice heterozygous or homozygous for the Kcne3-lacZ allele are fully viable and fertile, and do not display overt vascular phenotypes (data not shown). Analysis of $\beta$-galactosidase activity in retinas of Kcne3-lacZ reporter mice at P7 revealed highly specific expression within the leading edge of the developing vascular plexus (Fig. 2a-c). Unlike Dll4, which is expressed by both ETCs and arterial endothelial cells [9, 16], Kcne3-lacZ remains restricted to ETCs and some ESCs of the angiogenic front but is absent from differentiated vessels (arteries, veins, hyaloid).

We next investigated Kcne3 gene expression under conditions of pathologic retinal angiogenesis. The widely used oxygen-induced retinopathy (OIR) murine model recapitulates pathogenic aspects of neovascular retinopathies in humans. Here, vascular degeneration and ischemia within the central retina is induced by exposure of early postnatal mice (P6-P11) to high oxygen levels (75\%), which upon return to normoxia manifest pathologic neovascular tuft invasion of the vitreal space-a phenomenon largely governed by dysregulated Vegfa expression [37]. To correlate between overt changes to vessel morphology and associated transcriptomic alterations under OIR conditions, at the beginning of neovascular tuft formation at P15, a single dose of hFc or VEGF-Trap was systemically delivered (Fig. 2d). Vessel analysis (IB4) and RNAseq were performed on retinal samples collected 6- and 24-h following hFc or VEGFTrap administration ( $N=3-5$ per group per time point). While retina from $\mathrm{hFc}$-injected mice exhibited prominent central vaso-obliteration and formation of neovascular tufts that increased in severity between baseline and 24-h (Fig. 2e, f), a smaller avascular area and an abatement of neovascular tuft formation was observed under VEGF-Trap conditions. 
Parallel interrogation of transcriptome profiles revealed that while the majority of endothelial-specific genes were not significantly influenced by the administered agents, the expression of Kcne 3 and $E s m l$ were notably downregulated at 6- and 24-h following VEGF-Trap treatment (Fig. 2g, supplemental Table 2). Direct examination of Kcne3-lacZ reporter activity under conditions of OIR further revealed that Kcne 3 is active in endothelial cells surrounding the avascular zone by the end of the hyperoxic phase, but its levels increase considerably by 6-days following the return to normal oxygen levels (Fig. 2h). Interestingly, co-detection of Kcne3-lacZ/IB4 shows that Kcne3 activity is predominantly restricted to ETCs at the vascular-avascular interface but is largely absent from neovascular tufts (Fig. 2i, j). Together, these observations indicate that Kcne 3 is a specific ETC marker during normal and pathologic retinal angiogenesis, which can further expand into adjacent endothelial cell types under VEGFA excess.

\section{Kcne3 is an ETC-specific gene during embryonic development}

To examine whether Kcne3 may be a broad ETC marker in additional angiogenic contexts, we investigated Kcne3-lacZ expression dynamics during successive stages of mouse embryogenesis. In mouse, embryonic sprouting angiogenesis initiates shortly following the vasculogenic formation of primary aortic and venous structures ( E7.5 to E8.5) [4, 38]. Comparatively, Kcne3-lacZ reporter activity first becomes evident at $\sim$ E9.0 within sparse $l a c Z^{+}$endothelial cells localizing to the facial and branchial prominences, intersomitic space, developing limb buds, and in juxtaposition to aspects of the neural tube (Fig. 3a-d). Interestingly, this nascent expression pattern significantly expands within 24-h (E10.5), where discernable Kcne3-LacZ-positive microvascular sprouts are present in the nasal prominence, branchial arches, limbs, hyaloid vessel of the eye, and portions of segmental vessels along the spinal column (Fig. 3e, g, h, $\mathrm{j}, \mathrm{k}$ ). As Kcne3 is responsive to VEGFA in the retina, we sought to correlate its expression to that of embryonic Vegfa (E10.5). Interestingly, Vegfa expression as determined by WMISH, localizes to mesenchymal cells within or adjacent to Kcne 3 + domains within the facial prominences, branchial arches, and limb buds (Fig. 3f, i, 1), as previously reported $[39,40]$. Discerning the identity of $\mathrm{Kcne}^{3}-\mathrm{lacZ}^{+}$cells, immunofluorescent analysis using anti- $\beta$-galactosidase and anti-CD31 (Pecam1)-specific antiserum shows that reporter activity is restricted to putative ETCs, but absent from the endothelium of lumenized vessels (Fig. 4a, b). Thus, Kcne 3 activation initiates within sprouting microvessels during early phases of embryonic angiogenesis and in close spatial and temporal association with Vegfa.
Interestingly, Vegfa originating from the primordial appendicular skeleton has been shown to critically regulate vascular morphogenesis in the limb [39]. Using RNAscope technology, double In Situ Hybridization analysis of Kcne3 and Pecaml in the developing distal limb at E13.5, a stage at which chondrocytes begin to differentiate within the condensing digital mesenchyme, shows that Kcne 3 is expressed by all Pecam ${ }^{+}$endothelial sprouts surrounding the digital anlagen (Fig. 4c-e). In agreement, Kcne3-lacZ activity is remarkably pronounced within microvascular ECs closely associated with all endochondral skeletal elements by E15.5 (Fig. 4f, g, i, j), which abundantly populate both perichondrial and trabecular layers of the forming long bones (Fig. 4j). Outside of the skeleton at this stage, ECspecific Kcne3-lac $Z^{+}$activity is also observed within the coronary microvasculature (Fig. $4 \mathrm{~h}, \mathrm{k}$ ), and capillaries of the esophagus, stomach, thymus, and dermis (Suppl. Fig. 2C, data not shown). Only rarely did we observe Kcne3-lac $Z^{+}$in lumenized arterioles or venules. Our data therefore indicates that during progressive stages of embryonic development Kcne 3 specifically localizes to ETCs within vascular beds undergoing active angiogenesis, but is never expressed by endothelial cells of established veins or arteries.

\section{Kcne3 is specific to ETCs in during wound healing and tumor growth}

As Kcne 3 is expressed by ETCs during normal development and is induced under oxygen deprivation, we also sought to investigate its activity within other models of pathogenic angiogenesis purportedly involving postnatal VEGFA function. The cornea serves as an excellent system for evaluating VEGFA-mediated pathogenic angiogenesis, where placement of sutures within the avascular corneal center promotes the invasion of blood and lymphatic vascular sprouts originating from preexisting vessels in the peripheral limbus [41, 42]. Applying this model to Kcne3-lacZ reporter mice, corneal tissues were examined by whole mount $\beta$-galactosidase staining 9-days following suture implantation. Strikingly, intense and specific lacZ+ staining was observed at the leading edge of sprouting vessels within close proximity of the sutures, however, no staining was noted in the limbal vasculature (Fig. 5a-c, arrows). The morphology and positioning of stained endothelial cells suggests that these are comprised mostly of ETCs and some ESCs.

We next asked whether Kcne 3 is also upregulated during tumor angiogenesis. For this purpose, we evaluated $\beta$-gal expression in Lewis Lung Cell (LLC) tumor allografts introduced subcutaneously to homozygous Kcne3-lac Z mice. In this model, all $\beta$-gal expression is attributed to Kcne3-lacZ reporter activity originating from host-derived cells invading the tumor. Gross inspection shows robust and specific Kcne3-lacZ activity within 
Fig. 3 Kcne 3 is an early ETC marker during embryonic angiogenesis. a-d Analysis of $\beta$-galactosidase activity in homozygous Kcne3-lacZ embryos at E9.0 (21 somites), showing expression in nascent endothelial sprouts of the emerging forelimb bud (a, c), branchial arches and nasal prominence (B, arrow), and close proximity to mid- and hind-brain structures (d arrow). e-l Comparative of Kcne3-lacZ reporter activity and Vegfa mRNA (WMISH) in embryos at E10.5. Kcne3-lacZ reporter is specific to presumptive ETCs and endothelial sprouts forming the hyaloid vessels (g), microvasculature in branchial arches and facial prominence (h), intersomitic vasculature (j), and $\operatorname{limb}(\mathbf{k})$. Vegfa is detected in mesenchyme of all cephalic structures (f, i), heart (f), and limb (I). $e$ eye, $l i$ limb, $h t$ heart, bal first branchial arch
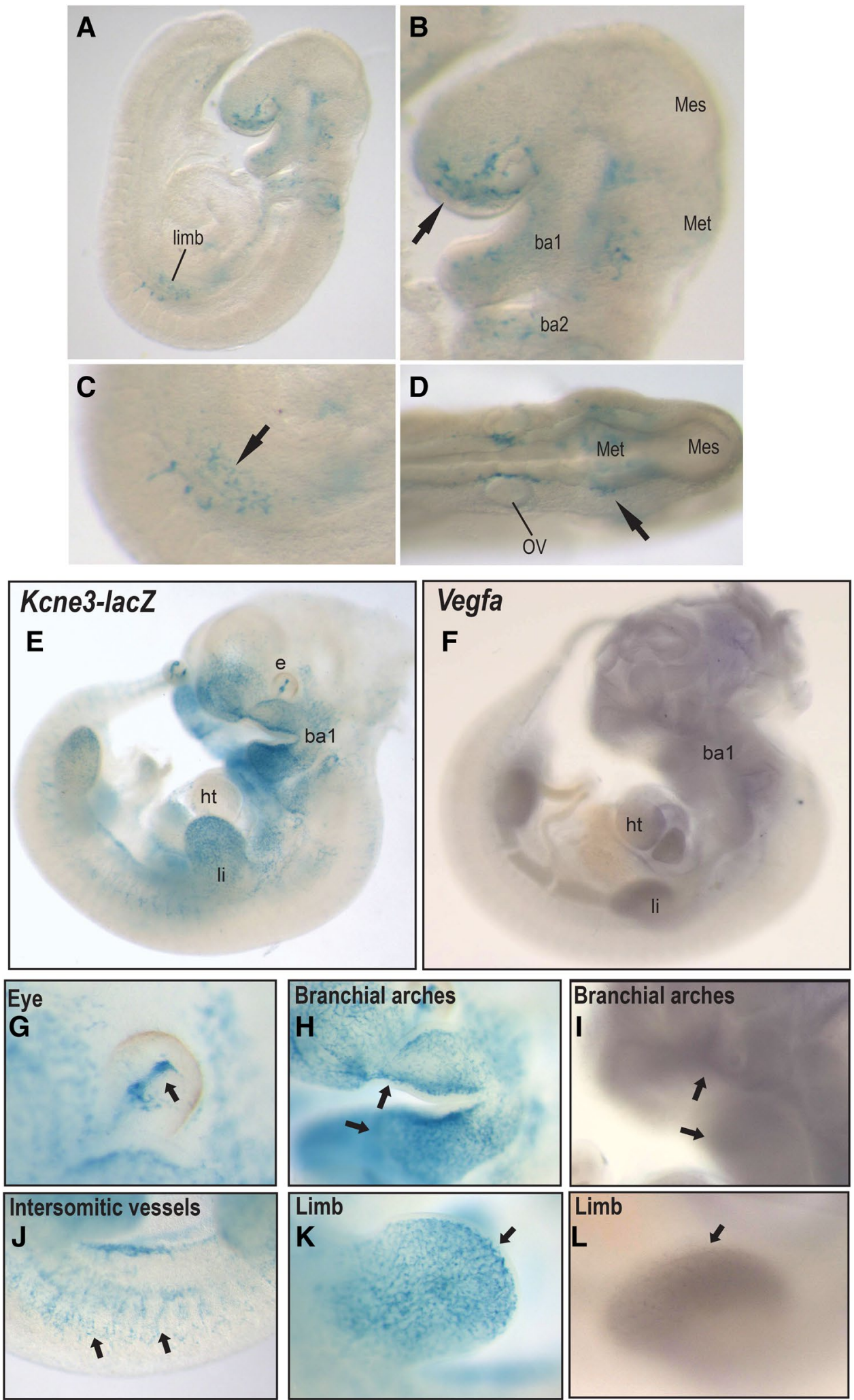

capillary sprouts and putative ETCs that primarily localize to the tumor perimeter (Fig. 5d). In contrast, LLC tumors grafted onto $l a c Z$ reporters with global endothelial activity, as shown for $K d r$-lacZ, exhibit broad capillary vessel staining that permeates the entire tumor (Fig. 5e). An additional tumor model we explored relies on the propensity of murine embryonic stem cells (ESCs) to form subcutaneous allograft teratomas, in which ESCs give rise 

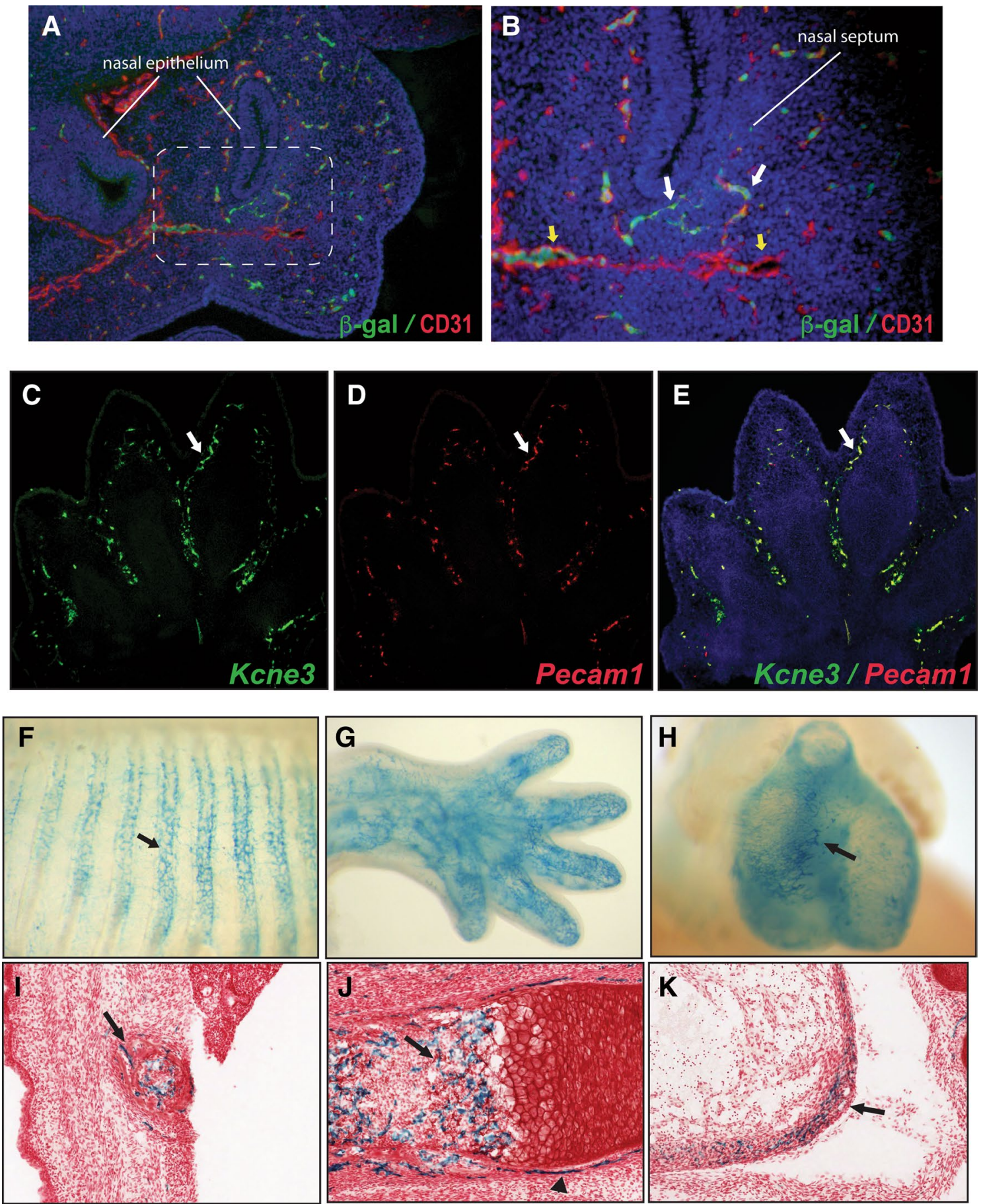

Fig. 4 Kcne 3 localizes to ECs associated with the developing skeleton. a, b Immunodetection of $\beta$-galactosidase (green) and CD31 (red) within the nasal prominence of a Kcne3-lacZ embryo at E10.5 ("b" is a high-power view of dotted area in "a"). Kcne3-lacZ $Z^{+}$ECs are detected in microvascular sprouts within the condensing mesenchyme of the nasal septum (white arrows $\mathbf{b}$ ), but are absent from lumenized vessels (yellow arrows b; Note: autofluorescent red blood cells are present in vessel lumen). c-e Double fluorescent In Situ Hybridization (ISH) employing RNAscope technology to simultaneously detect Kcne3 (green) and Pecaml (red) mRNA in the distal limb of a wild-type mouse embryo at E13.5. Kcne3 specifically localizes to all Pecaml ${ }^{+}$ECs surrounding the cartilaginous digital elements. $\mathbf{f}-\mathbf{k} \beta$-galactosidase expression in Kcne3-lacZ mice at E15.5 showing robust staining in microvascular ECs surrounding skeletal anlagen including ribs (f, i i), distal limb (g), and femur (j). At this stage, Kcne3-LacZ ${ }^{+}$ECs are also in the coronary microvasculature of the heart $(\mathbf{h}, \mathbf{k})$ 

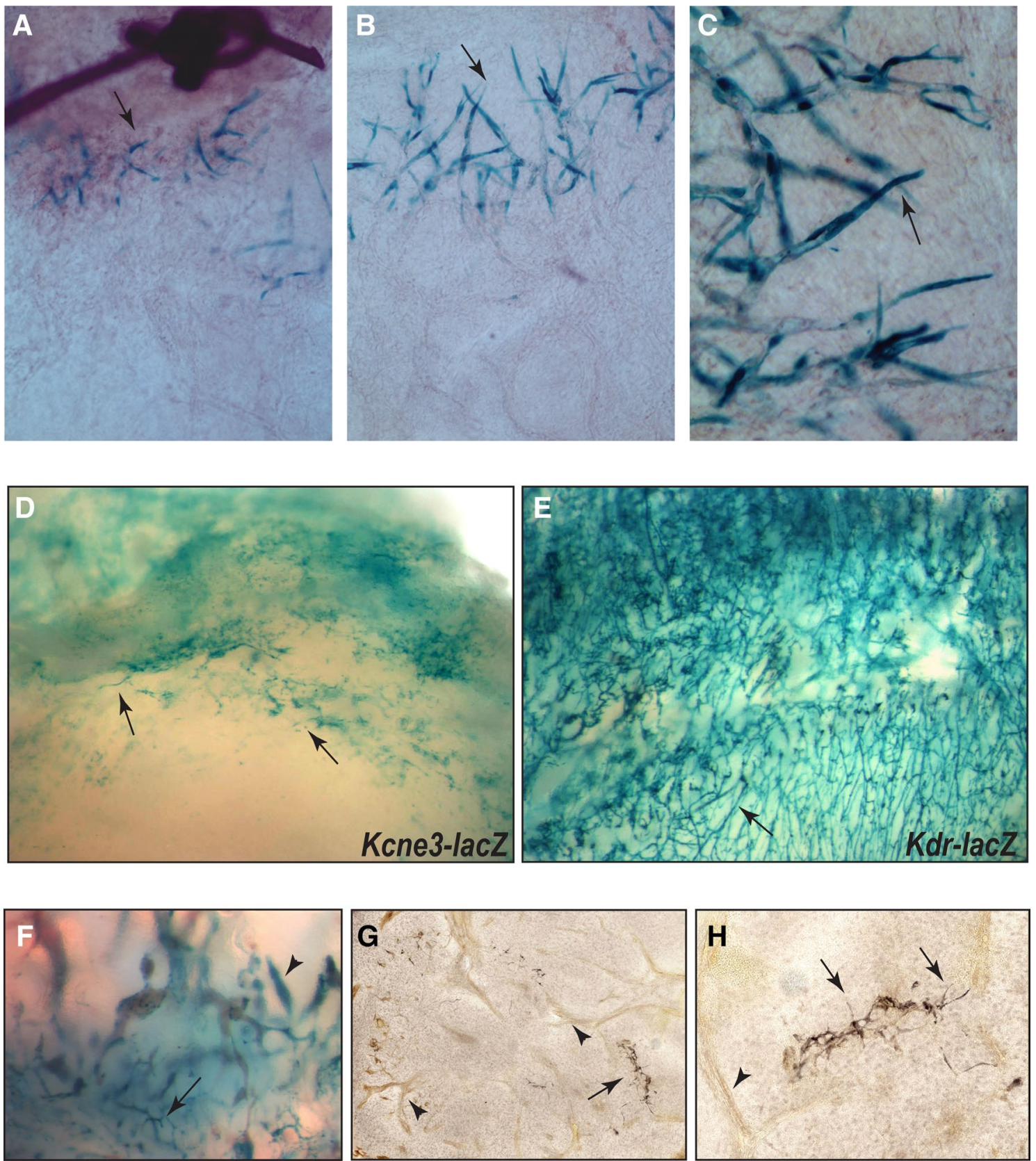

Fig. 5 Kcne 3 is expressed by endothelial tip cells during pathogenic angiogenesis. a-c Kcne3-lacZ reporter expression in the corneal suture injury model. Seven days after suture insertion into the central cornea of Kcne3-lacZ reporter mice, $\beta$-galactosidase is detected in ETCs immediately adjacent to the suture implantation site (arrows), but not in any other vascular structures emanating from the limbal arcade. d LLC cells allografted subcutaneously into Kcne3-lacZ homozygous mice result in prominent host-derived lacZ expression within edges of the tumor microvasculature (d, arrows), but not in

to multiple cell lineages and tissue types [33]. Interestingly, ESC-derived endothelial cells have been shown to incorporate and contribute to the formation of the teratoma vasculature [33]. Allografted ESC clones harboring larger venules or arterioles. e LLC tumor allograft in $K d r$-lacZ mouse showing broad expression throughout the capillary network (arrow). f Subcutaneous teratomas derived from Kcne3-lacZ ES cells showing specific $\beta$-galactosidase staining in microvascular (arrow) and epithelial structures (arrowhead). $\mathbf{g}, \mathbf{h}$ Low- and high-power images of sectioned Kcne3-lacZ ES tumors showing the immuno-detection of CD31 in endothelial cells of lumenized blood vessels and capillaries (brown, arrowheads), while $\beta$-galactosidase is expressed solely by CD $31^{+}$ETCs (black, arrows)

the Kcne3-lac Z allele into WT C57BL6 mice generated heterogeneous tumors that upon $\beta$-galactosidase staining revealed two discernable lacZ ${ }^{+}$tissue types: epithelial and endothelial (Fig. 5f). Close examination of the 
tumor vasculature, however, shows that Kcne3-lacZ is specifically activated in endothelial cells localized to the microvasculature within peripheral aspects of the teratoma, which are revealed to be CD $31^{+}$ESC-derived ETCs (Fig. 5g, h). Notably, Kcne3-lacZ reporter activity was conspicuously absent from endothelial cells of lumenized vessels. Taken together, our data indicates that Kcne 3 is also a specific ETC marker during tumor angiogenesis.

\section{Expression of Kcne3 in non-vascular tissues and organs}

While initiating in embryonic microvascular endothelial cells, starting at E13.5 we also detected Kcne 3 mRNA in the gut epithelium by WMISH (Suppl. Fig. 2A, B). Indeed, examination of Kcne3-lacZ reporter mice shows that in addition to the intestine, expression prominently expands into the mucosal epithelial layers of the esophagus and stomach by E15.5 (Suppl. Fig. 2C-E, data not shown). By comparison to fetal stages, epithelial Kcne3-lacZ becomes more pronounced in adult organs. In agreement with prior reports, a cross-tissue examination at age of 12 weeks shows strong expression within the crypts of the small intestinal villi, and in the entire mucosal layer of the large intestine (Suppl. Fig. 3A, $\mathrm{B}$; data not shown). In addition, however, our analysis reveals expression within organs and cell types not yet described. Surprisingly, robust epithelial Kcne3-lacZ staining is detected throughout the liver bile duct network, which further extends into the gallbladder proper (Suppl. Fig. 3B-F). Additional epithelial expression is observed within the trachea, salivary gland, lacrimal ducts, cutaneous sebaceous glands, pancreatic ducts (Suppl. Fig. 3G, H; data not shown). Although not characterized histologically, specific staining within mesenteric lymph nodes is suggestive of expression in immune cell types (Suppl. Fig. 3I). In addition, female mice exhibit distinct Kcne3-lacZ activity in ovaries and the oviduct (Suppl. Fig. 3J).

Consistent with expression in ETCs, which are largely absent in normal mature tissues, analysis of adult organs shows that by comparison to embryos, only a limited number of vascular beds exhibit Kcne3-lacZ expression. Here we observed expression in the arcuate arteries of the kidney, mesenteric vasculature, and peripheral arterioles within muscle (Suppl. Fig. 3K-N). Punctate lacZ staining is also present in growth plates of endochondral bone, but to a much lesser extent than observed in the fetal skeleton (Suppl. Fig. 3O, P). Thus, although Kcne3 initiates as an ETC-specific gene during embryonic and retinal development, its postnatal extravascular expression likely reflects broader physiological functions.

\section{Discussion}

A number of findings presented in this study indicate that, within the vasculature, Kcne 3 is a VEGFA-inducible gene selectively expressed by endothelial tip cells during normal and pathogenic angiogenesis. First, our transcriptome and spatial analyses of Kcne 3 localization within the retinal vascular plexus are consistent with published microarray data identifying Kcne 3 amongst genes upregulated in retinal ETCs [21]. Second, reporter allele activity confirms that Kcne 3 is specific to ETCs during developmental retinal angiogenesis, neovascular recovery following OIR, corneal injury, and in several tumor angiogenic models. While we postulate that ETC restriction of Kcne 3 is primarily regulated by astrocyte-sourced VEGFA during developmental retinal angiogenesis [6], a pattern also maintained along the vaso-obliterative perimeter in OIR, it is conspicuously excluded from pathologic neovascular tufts. Although the precise mechanism of neovascular tuft formation is not entirely clear, these consist of highly disorganized, poorly perfused, small-caliber vessels that lack identifiable tip cells [15]. It is therefore likely that Kcne 3 localizes to pre-tuft ETCs but is not induced within pathogenic non-tip endothelial cells, even in the presence of elevated VEGFA. Nonetheless, total VEGFA-blockade at this critical juncture both normalizes pathogenic angiogenesis and rapidly suppresses Kcne 3 transcription (most likely in bone fide ETCs).

Remarkably, intravitreal delivery of VEGFA during physiological retinal angiogenesis results in robust and immediate upregulation of Kcne 3 within the majority of ECs comprising the retinal vascular plexus (excluding those populating differentiated vessels), suggesting a broad potential by endothelial cells to respond to VEGFA. This is not entirely unexpected since ETC identity and phenotype, including filopodia formation and directionality, are dictated by fine VEGFA gradients emanating from concentric astrocytes [6]; overriding these by VEGFA excess, results in altered ETC distribution, filopodia misguidance, and overt alterations to vasoconstriction [6,34]. Although our data strongly suggests that Kcne 3 is a VEGFA-inducible gene, additional molecular studies will be necessary to determine whether it is a direct or indirect target of VGEFA-VEGFR2 signaling in ECs. VEGFA-mediated receptor tyrosine kinase activation transduces multiple complex effector pathways, however, their associated transcription factors (e.g. SoxF, EST1) have only recently been recognized [52, 53]. Interestingly, a recent study identified Kcne3 (and Esm I) amongst the top FoxO-regulated genes downstream of PI3-AKT, an effector pathway of VEGFR2, in lung and liver ECs [54]. Whether Kcne 3 is in fact regulated by FoxO1/3/4 in cis remains a compelling area of 
investigation that may provide answers on how this gene is transcriptionally regulated by VEGFA signal outputs.

In agreement, the temporal and spatial expression dynamics of Kcne 3 closely mimic that of Esml, but not of other purported tip-cell genes (e.g. Dll4, Angpt2, Cxcr4). Esml was initially identified as an endothelial-specific VEGFA target gene [43, 44], which in the retina is restricted to ETCs and in turn potentiates several aspects of VEGFA ${ }_{165}$ bioactivity [19, 20, 23]. Thus, both Kcne 3 and Esml have been shown to be similarly suppressed in tumors responding to VEGFA-blockade [45]. However, in contrast to Kcne3, which appears to be a universal ETC marker in multiple angiogenic contexts (retina, embryo, tumor), Esml loses ETC-specificity outside of the retina [20]. In fact, during embryonic angiogenesis, Kcne3-lacZ and mRNA closely apposes Vegfa expression domains-most notably in the developing limb and endochondral skeletal elements. This latter aspect is of particular interest as VEGFA production by the chondrogenic lineage is crucial for primordial limb angiogenesis [39], and the subsequent regulation of fetal and postnatal cartilage and bone formation [46-49]. The recent identification of a molecularly distinct EC subtype that supports osteoblastogenesis and matrix mineralization is consistent with Kcne3 + ECs tightly associating with metaphyseal ossification centers, and thus may serve as a specific VEGFA-responsive and targetable marker for this unique cell population [50,51]. Thus, in multiple contexts Kcne 3 shows characteristics of a bone fide ETC marker that is highly attuned to local VEGF-ligand concentrations.

Consistent with previously published data [26, 27], we find that homozygous $K c n e 3^{\text {lacZ/lacZ }}$ are fully viable and do not manifest overt phenotypes under non-challenged conditions. Excluding the possibility of subtle changes to angiogenesis, Kcne 3 mutants did not display obvious alterations to embryonic or retinal vessel morphology (not shown), suggesting that this gene is not required for vascular development. However, whether a functional role for Kcne3 may be revealed under pathogenic neovascular settings remains to be investigated. The four classes of potassium channels comprised of 77 genes and 16 families, of which many associate with additional accessory $\beta$-subunits (e.g. Kcne3), ensures immense diversity and probable functional redundancy [55]. Recently, a functional role for the inwardly-rectifying potassium channel Kcnj2/Kir2.1 has been demonstrated in retinal vascular remodeling [56]. It is possible that a clearer biological role for Kcne 3 in endothelial cells would be elucidated with the combinatorial ablation of its primary channel components, Kcnq1 or Kcnc4. Alternatively, Kcne3 may exert gain-of-function effects under conditions of ectopic overexpression, as mediated by VEGFA excess. In agreement, ectopic expression of Kcne3 in the heart was shown to alter the electrophysiological properties of ventricular contraction [57].
Beyond endothelial activation, our study revealed that Kcne 3 is subsequently expressed by multiple epithelial cell types. While Kcne3-lacZ reporter activity in the alimentary system (esophagus, stomach, intestine) is consistent with prior reports [26], its expression within hepatic bile duct network and gallbladder is suggestive of broader roles in maintaining electrophysiological properties across mucosal membranes. Hence, while we propose that the Kcne 3 allele can be effectively engineered for fate mapping the ETC lineage, as demonstrated for Esml [23, 58], careful strategies should be considered when pursuing ETC-specific loss-offunction studies.

Acknowledgements We dedicate this manuscript to the memory of Pat Boland, whose work significantly contributed to this study.

Open Access This article is distributed under the terms of the Creative Commons Attribution 4.0 International License (http://creativeco mmons.org/licenses/by/4.0/), which permits unrestricted use, distribution, and reproduction in any medium, provided you give appropriate credit to the original author(s) and the source, provide a link to the Creative Commons license, and indicate if changes were made.

\section{References}

1. Carmeliet P, Jain RK (2000) Angiogenesis in cancer and other diseases. Nature 407(6801):249-257

2. Carmeliet P, Jain RK (2011) Molecular mechanisms and clinical applications of angiogenesis. Nature 473(7347):298-307

3. Duh EJ, Sun JK, Stitt AW (2017) Diabetic retinopathy: current understanding, mechanisms, and treatment strategies. JCI Insight 2(14):e93751

4. Potente M, Gerhardt H, Carmeliet P (2011) Basic and therapeutic aspects of angiogenesis. Cell 146(6):873-887

5. Roca C, Adams RH (2007) Regulation of vascular morphogenesis by Notch signaling. Genes Dev 21(20):2511-2524

6. Gerhardt $\mathrm{H}$ et al (2003) VEGF guides angiogenic sprouting utilizing endothelial tip cell filopodia. J Cell Biol 161(6):1163-1177

7. Hellstrom M et al (2007) D1l4 signalling through Notch1 regulates formation of tip cells during angiogenesis. Nature 445(7129):776-780

8. Siekmann AF, Lawson ND (2007) Notch signalling limits angiogenic cell behaviour in developing zebrafish arteries. Nature 445(7129):781-784

9. Lobov IB et al (2007) Delta-like ligand 4 (D114) is induced by VEGF as a negative regulator of angiogenic sprouting. Proc Natl Acad Sci USA 104(9):3219-3224

10. Suchting $\mathrm{S}$ et al (2007) The Notch ligand Delta-like 4 negatively regulates endothelial tip cell formation and vessel branching. Proc Natl Acad Sci USA 104(9):3225-3230

11. Ehling $\mathrm{M}$ et al (2013) Notch controls retinal blood vessel maturation and quiescence. Development 140(14):3051-3061

12. Carmeliet P et al (1996) Abnormal blood vessel development and lethality in embryos lacking a single VEGF allele. Nature 380(6573):435-439

13. Ferrara $\mathrm{N}$ et al (1996) Heterozygous embryonic lethality induced by targeted inactivation of the VEGF gene. Nature 380(6573):439-442

14. Gale NW et al (2004) Haploinsufficiency of delta-like 4 ligand results in embryonic lethality due to major defects in 
arterial and vascular development. Proc Natl Acad Sci USA 101(45):15949-15954

15. Stahl A et al (2010) The mouse retina as an angiogenesis model. Invest Ophthalmol Vis Sci 51(6):2813-2826

16. Claxton S, Fruttiger M (2004) Periodic Delta-like 4 expression in developing retinal arteries. Gene Expr Patterns 5(1):123-127

17. Lu X et al (2004) The netrin receptor UNC5B mediates guidance events controlling morphogenesis of the vascular system. Nature 432(7014):179-186

18. Tammela $\mathrm{T}$ et al (2008) Blocking VEGFR-3 suppresses angiogenic sprouting and vascular network formation. Nature 454(7204):656-660

19. del Toro $\mathrm{R}$ et al (2010) Identification and functional analysis of endothelial tip cell-enriched genes. Blood 116(19):4025-4033

20. Rocha SF et al (2014) Esm1 modulates endothelial tip cell behavior and vascular permeability by enhancing VEGF bioavailability. Circ Res 115(6):581-590

21. Strasser GA, Kaminker JS, Tessier-Lavigne M (2010) Microarray analysis of retinal endothelial tip cells identifies CXCR4 as a mediator of tip cell morphology and branching. Blood 115(24):5102-5110

22. Nowak-Sliwinska P et al (2018) Consensus guidelines for the use and interpretation of angiogenesis assays. Angiogenesis 21(3):425-532

23. Pitulescu ME et al (2017) Dll4 and Notch signalling couples sprouting angiogenesis and artery formation. Nat Cell Biol 19(8):915-927

24. Abbott GW (2016) KCNE1 and KCNE3: The yin and yang of voltage-gated $\mathrm{K}(+)$ channel regulation. Gene 576(1 Pt 1):1-13

25. Abbott GW et al (2001) MiRP2 forms potassium channels in skeletal muscle with $\mathrm{Kv} 3.4$ and is associated with periodic paralysis. Cell 104(2):217-231

26. Preston $\mathrm{P}$ et al (2010) Disruption of the $\mathrm{K}+$ channel beta-subunit KCNE3 reveals an important role in intestinal and tracheal $\mathrm{Cl}^{-}$ transport. J Biol Chem 285(10):7165-7175

27. $\mathrm{Hu} \mathrm{Z}$ et al (2014) Kcne3 deletion initiates extracardiac arrhythmogenesis in mice. FASEB J 28(2):935-945

28. Valenzuela DM et al (2003) High-throughput engineering of the mouse genome coupled with high-resolution expression analysis. Nat Biotechnol 21(6):652-659

29. Adams NC, Gale NW (2006) High resolution gene expression analysis in mice using genetically inserted reporter genes. In: Pease S, Lois C (eds) Mammalian and avian transgenesis-new approaches. Springer, Berlin

30. Wang F et al (2012) RNAscope: a novel in situ RNA analysis platform for formalin-fixed, paraffin-embedded tissues. J Mol Diagn 14(1):22-29

31. Smith LE et al (1994) Oxygen-induced retinopathy in the mouse. Invest Ophthalmol Vis Sci 35(1):101-111

32. Cursiefen $\mathrm{C}$ et al (2004) Inhibition of hemangiogenesis and lymphangiogenesis after normal-risk corneal transplantation by neutralizing VEGF promotes graft survival. Invest Ophthalmol Vis Sci 45(8):2666-2673

33. Li Z et al (2009) Embryonic stem cell tumor model reveals role of vascular endothelial receptor tyrosine phosphatase in regulating Tie2 pathway in tumor angiogenesis. Proc Natl Acad Sci USA 106(52):22399-22404

34. Lobov IB et al (2011) The Dll4/Notch pathway controls postangiogenic blood vessel remodeling and regression by modulating vasoconstriction and blood flow. Blood 117(24):6728-6737

35. Holash J et al (2002) VEGF-Trap: a VEGF blocker with potent antitumor effects. Proc Natl Acad Sci USA 99(17):11393-11398

36. Zhao Q et al (2018) Single-cell transcriptome analyses reveal endothelial cell heterogeneity in tumors and changes following antiangiogenic treatment. Cancer Res 78(9):2370-2382

37. Kim CB, D'Amore PA, Connor KM (2016) Revisiting the mouse model of oxygen-induced retinopathy. Eye Brain 8:67-79
38. Coultas L, Chawengsaksophak K, Rossant J (2005) Endothelial cells and VEGF in vascular development. Nature 438(7070):937-945

39. Eshkar-Oren I et al (2009) The forming limb skeleton serves as a signaling center for limb vasculature patterning via regulation of Vegf. Development 136(8):1263-1272

40. Miquerol L et al (1999) Multiple developmental roles of VEGF suggested by a LacZ-tagged allele. Dev Biol 212(2):307-322

41. Cursiefen C et al (2004) VEGF-A stimulates lymphangiogenesis and hemangiogenesis in inflammatory neovascularization via macrophage recruitment. J Clin Invest 113(7):1040-1050

42. Streilein JW et al (1996) Immunosuppressive properties of tissues obtained from eyes with experimentally manipulated corneas. Invest Ophthalmol Vis Sci 37(2):413-424

43. Rennel E et al (2007) Endocan is a VEGF-A and PI3K regulated gene with increased expression in human renal cancer. Exp Cell Res 313(7):1285-1294

44. Shin JW, Huggenberger R, Detmar M (2008) Transcriptional profiling of VEGF-A and VEGF-C target genes in lymphatic endothelium reveals endothelial-specific molecule- 1 as a novel mediator of lymphangiogenesis. Blood 112(6):2318-2326

45. Eichten A et al (2013) Rapid decrease in tumor perfusion following VEGF blockade predicts long-term tumor growth inhibition in preclinical tumor models. Angiogenesis 16(2):429-441

46. Gerber HP et al (1999) VEGF couples hypertrophic cartilage remodeling, ossification and angiogenesis during endochondral bone formation. Nat Med 5(6):623-628

47. Maes C et al (2004) Soluble VEGF isoforms are essential for establishing epiphyseal vascularization and regulating chondrocyte development and survival. J Clin Invest 113(2):188-199

48. Zelzer E et al (2004) VEGFA is necessary for chondrocyte survival during bone development. Development 131(9):2161-2171

49. Zelzer E et al (2002) Skeletal defects in $\operatorname{VEGF(120/120)~mice~}$ reveal multiple roles for VEGF in skeletogenesis. Development 129(8):1893-1904

50. Kusumbe AP, Ramasamy SK, Adams RH (2014) Coupling of angiogenesis and osteogenesis by a specific vessel subtype in bone. Nature 507(7492):323-328

51. Ramasamy SK et al (2014) Endothelial Notch activity promotes angiogenesis and osteogenesis in bone. Nature 507(7492):376-380

52. Chen J et al (2017) VEGF amplifies transcription through ETS1 acetylation to enable angiogenesis. Nat Commun 8(1):383

53. Kim K et al (2016) SoxF transcription factors are positive feedback regulators of VEGF signaling. Circ Res 119(7):839-852

54. Paik JH et al (2007) FoxOs are lineage-restricted redundant tumor suppressors and regulate endothelial cell homeostasis. Cell 128(2):309-323

55. Pardo LA, Stuhmer W (2014) The roles of K(+) channels in cancer. Nat Rev Cancer 14(1):39-48

56. Boriushkin E, Fancher IS, Levitan I (2019) Shear-stress sensitive inwardly-rectifying $\mathrm{K}(+)$ channels regulate developmental retinal angiogenesis by vessel regression. Cell Physiol Biochem 52(6):1569-1583

57. Mazhari R et al (2002) Ectopic expression of KCNE3 accelerates cardiac repolarization and abbreviates the QT interval. J Clin Invest 109(8):1083-1090

58. $\mathrm{Xu} \mathrm{C}$ et al (2014) Arteries are formed by vein-derived endothelial tip cells. Nat Commun 5:5758

Publisher's Note Springer Nature remains neutral with regard to jurisdictional claims in published maps and institutional affiliations. 